\title{
Analysis of the electrolytically polished skeletal dentures surfaces using various nano- and microscopic technologies
}

\author{
TOMASZ DABROWA ${ }^{1 *}$, WOJCIECH MAJSTRZYK ${ }^{2}$, MAGDALENA TAMULEWICZ $^{2}$, \\ TOMASZ PIASECKI ${ }^{2}$, PIOTR KUNICKI ${ }^{2}$, WŁODZIMIERZ WIĘCKIEWICZ ${ }^{1}$, TEODOR GOTSZALK ${ }^{2}$ \\ ${ }^{1}$ Department of Prosthodontics, Wroclaw Medical University, Wrocław, Poland. \\ ${ }^{2}$ Faculty of Microsystem Electronics and Photonics, Wrocław University of Science and Technology, Wrocław, Poland.
}

\begin{abstract}
Purpose: The surface roughness of the dental restorations is significant to the denture plaque adhesion. Methods: In this work, we present the complex analysis of the electropolished $\mathrm{CoCrW}$ alloy remanium ${ }^{\circledR}$ star (Dentaurum, Germany) samples with laserengraved fiducial marks performed using complementary set of micro- and nanoscopic techniques: optical profilometry (OP), atomic force microscopy (AFM), scanning electron microscopy (SEM) and focused ion beam (FIB) milling. Results: Both mean and RMS roughness of the samples were reduced by electopolishing process, however, the results obtained using OP and AFM exhibited some discrepancies. This was caused by the relatively high local protruding defects developed on the processed surface. The cross-sections of the protrusions were made to analyze the cause of their formation as the EDS elemental content maps revealed that their composition was uniform. We also analyzed the local roughness in the smaller areas free from the defects. Conclusions: In that case, both OP and AFM techniques delivered the same results. Analysis of results showed that various methods used for the surface roughness evaluation have to be used simultaneously to obtain complete and true analysis of the technological CoCrW samples.
\end{abstract}

Key words: surface roughness, scanning electron microscopy, removable partial denture, scanning probe microscopy, optical profilometry

\section{Introduction}

Electrolytic polishing is carried out in the first stage of processing the castings, made on the base of metal alloys. This method enables us to smoothen the surface of future dentures made of chromium-cobalt alloys. Thus, it reduces the likelihood of unfavourable stresses occurring or contamination of the alloy surface with particles of polishing materials. The metal casting process affects the surface roughness $\left(R_{a}\right)$ of the alloy. The recesses formed on its outer layer increase its porosity, thus causing greater adhesion of the biofilm to the surface of the prosthesis. The correctness of operation during the dental alloys processing have an impact on their corrosion resistance. The technological process and mandatory be- haviour of dental alloy processing regimes also have an impact on the strength, precision and fitting of the dentures made of them. The preparatory procedure for electrochemical treatment (electropolishing) is based on sanding and grinding, during which the surface of the alloy undergoes plastic deformation. As a result of friction and temperature, a thin layer is formed, referred to as a surface crumple. It has a damaged crystalline structure and finely divided metallic grains. Due to the final electropolishing, this layer is removed, which leads to an increase in the hardness of the alloy and a reduction in the coefficient of friction [1]. This improves the mechanical properties of retention elements in prostheses, in which precise elements such as telescopic crowns or other types of anchorages have been used. It also reduces the risk of premature wear of the latches surface, and enables

\footnotetext{
* Corresponding author: Tomasz Dąbrowa, Department of Prosthodontics, Wroclaw Medical University, ul. Krakowska 26, 50-425 Wrocław, Poland. Phone: 00487178402 91, e-mail: tomasz.dabrowa@umed.wroc.pl

Received: July 8th, 2019

Accepted for publication: October 11th, 2019
} 
the retention force of the prosthesis to remain constant over a longer period of time. It is also important to prepare the future prosthesis in such a way as to minimize the risk of microbial deposition on its surface. It should be remembered that such anchorages have microretences, which can be a place willingly populated by various types of bacteria and fungi. These places are difficult to clean by elderly and senile patients with limited motor skills and poor eyesight, who are using dental prostheses. This fact is important for maintaining the oral hygiene of prostheses and mouth at an appropriate level, thus reducing the risk of oral diseases.

Electropolishing is carried out after mechanical pre-treatment. During polishing, the object is placed in the electrolyte, which may be ethylene glycol, sulphuric acid or hydrochloric acid, and connected to the anode. The cathode is a plate of steel, lead or copper. Both electrodes are immersed in the electrolyte to form an electric circuit. On the roughness peaks, the current intensity is higher, which causes dissolving the metal and smoothing the surface of the material.

Based on literature data, our own thoughts and observation of selected areas of the remanium ${ }^{\circledR}$ star (Dentaurum, Germany) [2] alloy surface, we proposed our own procedure. It allows for a detailed analysis of phenomena occurring on the surface of the material in an accurate and repeatable manner. In our opinion, this should be used as a standard procedure.

Up to now, researchers have mostly characterized surface topography of the fixed and removable dentures using profilometry [3], optical profilometry [4], scanning electron microscopy (SEM) [5], [6] or atomic force microscope (AFM) [7]. The single line profilometers usually analyse the surface parameters based on a non-scanning principle. Moreover, in the surface analysis, the probe shape must be taken into the consideration and the calculation of the surface properties with the resolution less than $30 \mathrm{~nm}$ is quite cumbersome. The optical 3D profilometers deliver surface images in $X Y Z$ directions with high throughput but the height information can only be achieved based on non-direct system calibration. The scanning electron microscopes make it possible to image the surface properties with very high resolution but the obtained results do not contain exact height information. The atomic force microscopy (AFM) enables surface characterization in $X Y Z$ directions with the local resolution of several nanometres. In the AFM investigations interactions between the microprobe and the surface are observed to rec- ord the surface topography. Very small tip radius and the feedback loop make it possible to determine the height of the surface features at each point on the investigated surface [8]. It must be also said that the AFM technology opens variety of possibilities to directly analyse the mechanical surface properties, so, from that point of view, its application in the research of the dental materials is fully justified.

Due to the progress in the experimental instrumentation, the aforementioned methods seem to be very attractive for the $\mathrm{CoCrW}$ surface characterization. In this work, we decided to compare the results of the surface quality evaluation obtained using optical profilometry and atomic force microscopy.

The AFM technology was mostly used in dental research to study the tissue, tooth and biofilm structure. Results of the measurements of the technological samples must be analysed with much care as none of the applied imaging methods can record the surface around the steep protrusions in the reliable way. The combination of complementary technologies enables us to acquire information about the surface topography [9].

Moreover, we analysed the structure properties the selected features on the surface using focused ion beam (FIB) technique. This technology, which was applied to fabricate the cross-sections of the structures, identified by the atomic force microscope, whose dimensions were of tens of nanometres. In this way, also the technologies described above were used in the complementary manner as only this way the thorough analysis of the surface properties is possible [10]. From the resolution point of view, methods applied for the surface analysis can be divided into microscopic or nanoscopic ones.

The novelty of our approach relies on combination of micro- and nanoscopic technologies, as, according to our experience, only this way complementary information about the sample at micro- and nanoscale can be gained. Moreover, in order to image the surface areas at the same location, we fabricated the, so called, fiducial marks in laser ablation technology, which made it possible to identify the surface areas in various measurements routines and after subsequent technological steps [11].

\section{Materials and methods}

AFM investigations were carried out on a commercial NanoMan VS microscope with NanoScope V controller (Veeco Instruments) with the Nanooworld 
RTESPW cantilevers fabricated (resonance frequency in the range from 264 to $342 \mathrm{kHz}$, stiffness ranging from 20 to $80 \mathrm{~N} / \mathrm{m}$ ). All the measurements were performed in ambient conditions. Surface topography was recorded in Tapping Mode (TM) with scan rate $0.2 \mathrm{~Hz}$. Scan area on raw samples was $70 \times 70 \mu \mathrm{m}$ while the measurements on the polished ones were performed in $50 \times 50 \mu \mathrm{m}$ scan field due to over-etching of the fiducial marks.

Profilometry measurements were performed by a SENSOFAR PLUAPEX system. PLu Apex's confocal metrology allows for high precision non-contact measurements of aspherical and flat surfaces with up to 50 $\mathrm{mm}$ measurement range in $\mathrm{Z}$ axis. A green LED is used as a light source.

SEM and focused ion beam (FIB) investigations were carried our using a Helios 600 dual beam system. To obtain the best resolution and best contrast of the surface imaging low acceleration voltage $(2 \mathrm{kV})$ was used. Elemental analysis were performer with Energy dispersive (EDS) x-ray spectroscopy. SEM/FIB analysis were performed in the areas as investigated using atomic force microscope.

Galvo G8T SOJO (Polska) was used for the tests. The tested materials were placed in a solution for the electrolytic polishing of dental alloys Cr-Co-Mo Elektrol 1 Chema - Elektromet (Poland) [12]. The following polishing conditions were used. The current was $2 \mathrm{~A}$. The temperature of the solution was maintained at $35^{\circ} \mathrm{C}$, and the polishing time was respectively $2,4,6$ and 8 minutes.

Fiducial marks were made with femtosecond laser made by TRUMPF (TrueMicro). Process parameters - wavelength $1030 \mathrm{~nm}$, pulse time $880 \mathrm{fs}$, repetition frequency $500 \mathrm{~Hz}$, average optic power $12 \mathrm{~W}$, BURST 3 mode, about 300 pulses in each mark, focal length $163 \mathrm{~mm}$.
The results obtained by the both profilometry and AFM were analysed statistically to provide roughness parameters [13]. The surface roughness $R_{a}$ provides the average discrepancy between the reference plane (which is modeled by the mean surface height) and absolute value of deviation from the mean plane (1)

$$
R_{a}=\frac{1}{N} \sum_{n=1}^{N}\left|z_{n}-\bar{z}\right|
$$

$R_{q}$, on the other hand, is computed over the square of differences between the mean plane and deviation from it. These roughness parameters describe the vertical differences only.

$$
R_{q}=\sqrt{\frac{1}{N} \sum_{n=1}^{N}\left(z_{n}-\bar{z}\right)^{2}} .
$$

For the data and image analysis Gwyddion software was applied. Gwyddion is a modular program for scanning probe microscopy (SPM) data visualization and analysis. It can be used for general height field and (greyscale) image processing, for instance for the analysis of profilometry data or thickness maps from imaging spectrophotometry. It provides a large number of data processing routines including all the standard statistical characterization, levelling and data correction, filtering or grain marking functions [14], [15].

\section{Results}

The surface of unprocessed samples as well as the samples electropolished for 2 and 4 minutes were analysed using optical profilometer (OP) and AFM using fiducial marks to locate the same spot on the sample
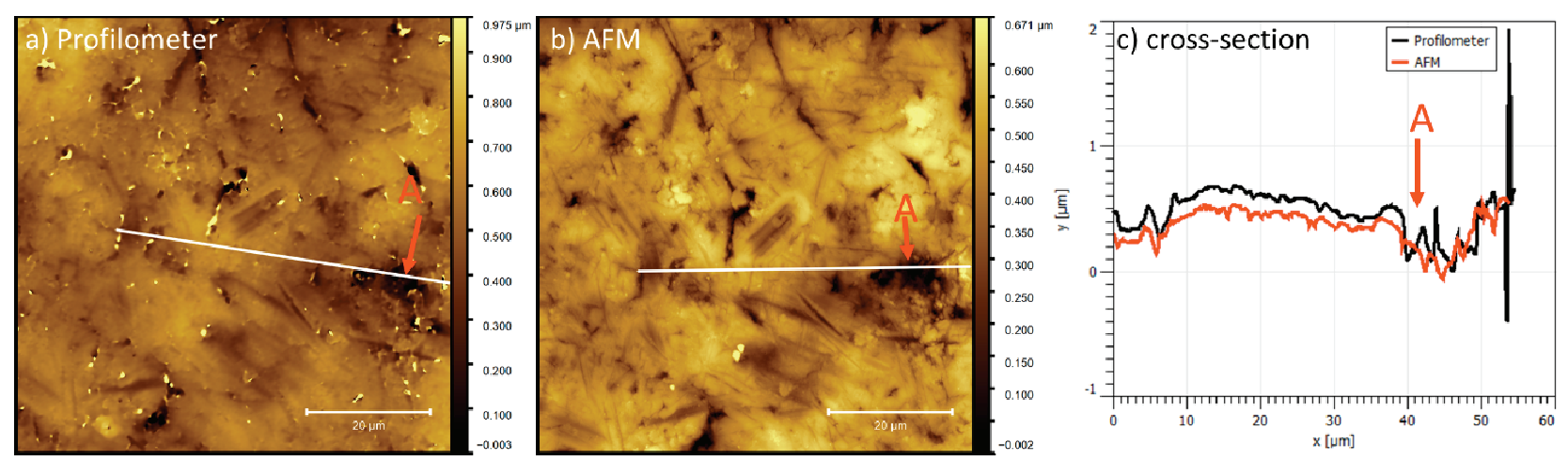

Fig. 1. Example surface of unprocessed samples seen using a) Profilometer and b) AFM. It is a representative set of images for all samples used before electropolishing.

The A-point marks the same feature of the corresponding images. c) The corresponding cross-section profiles from the Profilometry and AFM images as marked by the red lines 

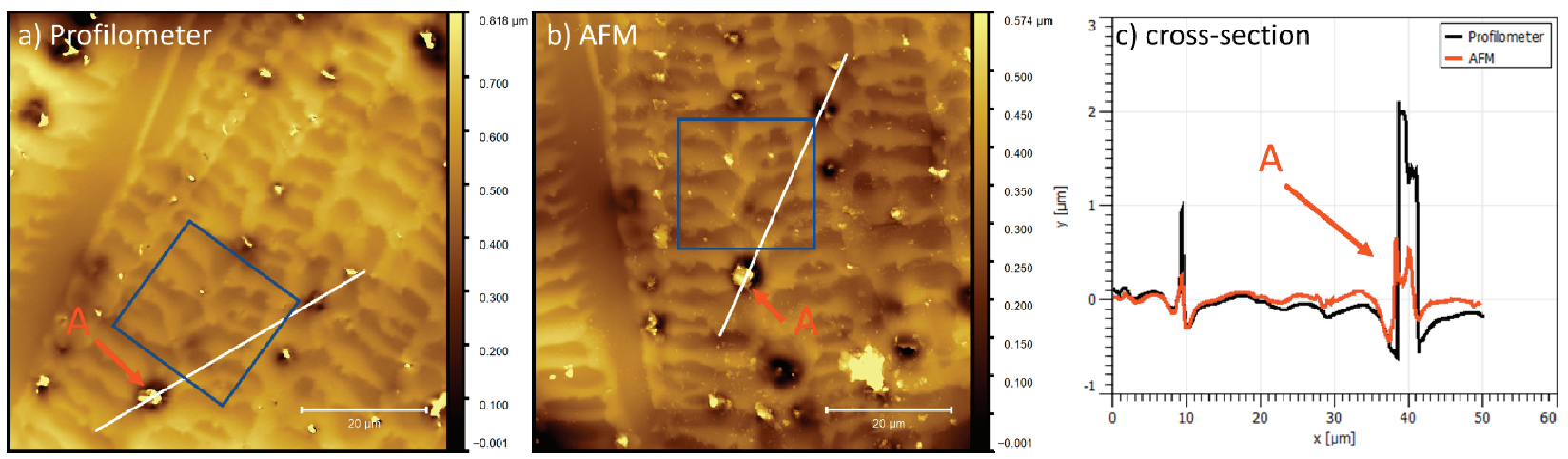

Fig. 2. The surface of the sample polished for 2 min seen using a) Profilometer and b) AFM.

The A-point marks the same feature of the corresponding images.

c) The corresponding cross-section profiles from the Profilometry and AFM images as marked by the red lines.

Notice that there is always a depression in which the protrusions are placed.

Aditionally the surface features regular "residual surface pattern".

The blue square indicates the region taken into considertion for local roughness estimation
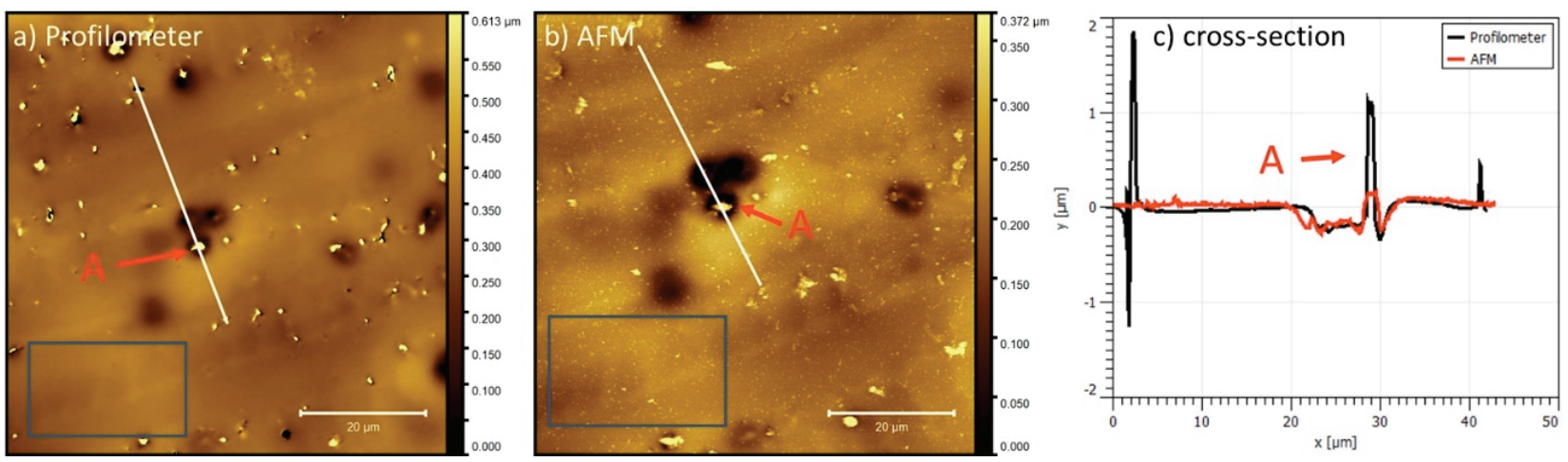

Fig. 3. The surface of the sample polished for 4 min seen using a) Profilometer and b) AFM.

The A-point marks the same feature of the corresponding images.

c) The corresponding cross-section profiles from the Profilometry and AFM images as marked by the red lines. Notice that the regular "residual surface pattern" completely disappeared, while the protrusions are still present.

The blue square indicates the region taken into considertion for local roughness estimation

Table 1. Global RMS and mean roughness calculated for samples before and after 2 min and 4 min of electropolishing process based on whole Atomic Force Microscope (AFM) and Optical Profilometer (OP) images

\begin{tabular}{|l|c|c|c|c|c|c|c|}
\hline \multirow{2}{*}{\multicolumn{1}{|c|}{ Value }} & \multirow{2}{*}{ Unit } & \multicolumn{2}{c|}{ Unprocessed } & \multicolumn{2}{c|}{2 min } & \multicolumn{2}{c|}{4 min } \\
\cline { 3 - 8 } & & AFM & OP & AFM & OP & AFM & OP \\
\hline Average value & $\mu \mathrm{m}$ & 0.6198 & 0.9624 & 0.4537 & 3.248 & 387.2 & 0 \\
\hline RMS roughness $\left(R_{q}\right)$ & $\mathrm{nm}$ & 95.75 & 127.8 & 77.87 & 151.6 & 42.59 & 103.0 \\
\hline Mean roughness $\left(R_{a}\right)$ & $\mathrm{nm}$ & 71.28 & 86.66 & 50.54 & 78.40 & 27.80 & 38.66 \\
\hline
\end{tabular}

Table 2. Local RMS and mean roughness calculated for samples before and after 2 min and 4 min of electropolishing process based on selected areas on the Atomic Force Microscope (AFM) and Optical Profilometer (OP) images

\begin{tabular}{|l|c|c|c|c|c|}
\hline \multirow{2}{*}{\multicolumn{1}{|c|}{ Value }} & \multirow{2}{*}{ Unit } & \multicolumn{2}{c|}{2 min } & \multicolumn{2}{c|}{4 min } \\
\cline { 3 - 6 } & & AFM & OP & AFM & OP \\
\hline Average value & $\mu \mathrm{m}$ & 458.9 & 3.204 & 404.9 & 21.77 \\
\hline RMS roughness $\left(R_{q}\right)$ & $\mathrm{nm}$ & 39.34 & 47.05 & 19.65 & 20.70 \\
\hline Mean roughness $\left(R_{a}\right)$ & $\mathrm{nm}$ & 29.56 & 32.65 & 14.68 & 17.18 \\
\hline
\end{tabular}




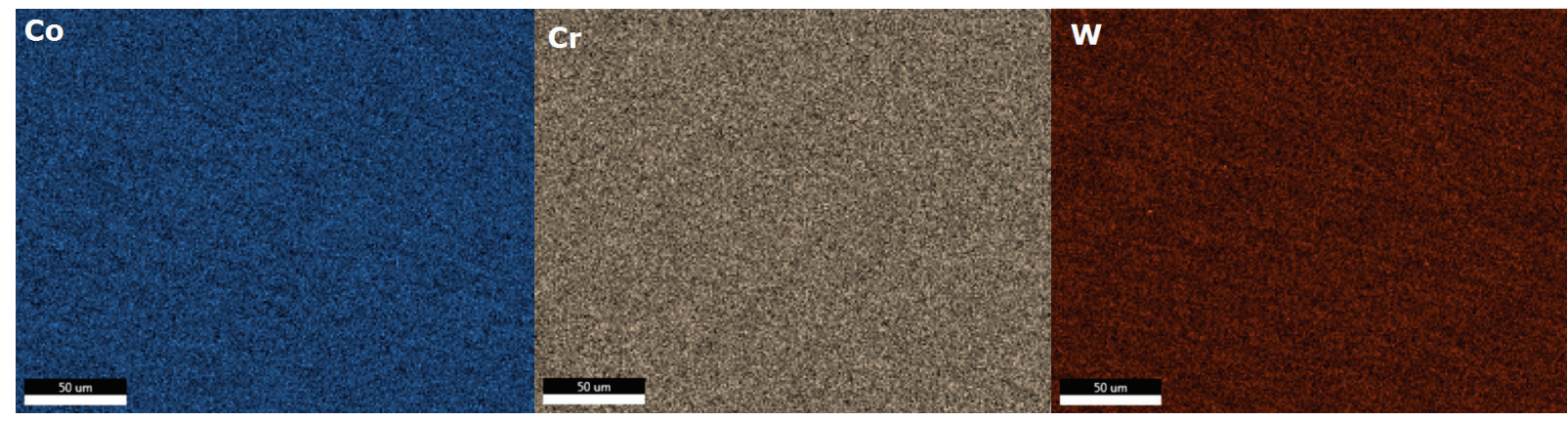

Fig. 4. EDS maps of elemental composition for cobalt (Co), chromium $(\mathrm{Cr})$ and tungsten (W)

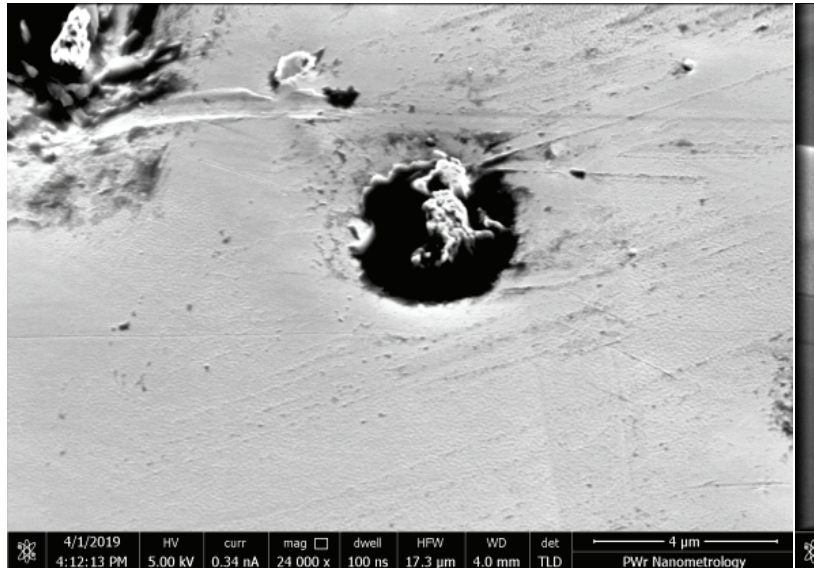

(a)

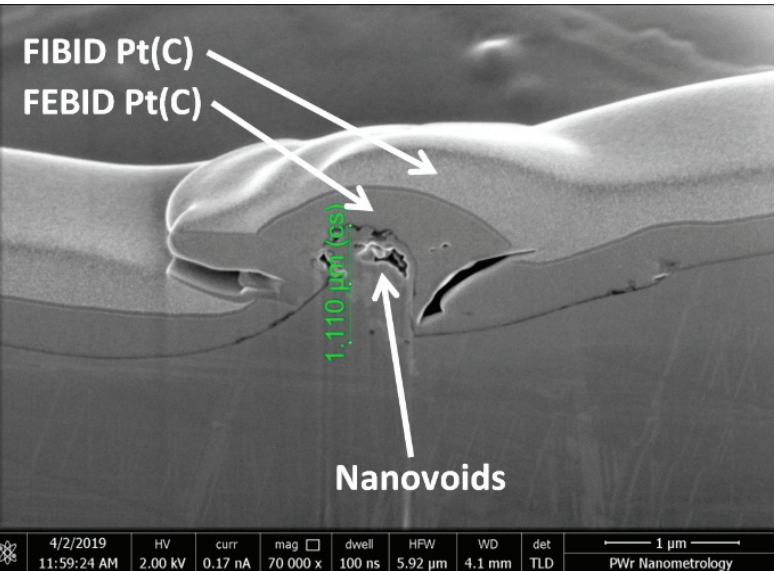

(b)

Fig. 5. Local protrusion of the sufrace of the electropolished samples: general view (a), the cross-section of the protrusion covered with FEBID and FIBID Pt(C), milled using FIB (b)

in each method. The scanning results as well as the cross-section made at the same locations are shown in Figs. 1-3.

Global RMS and mean roughness were calculated for images obtained using OP and AFM. The results are shown in Table 1.

On the surface of the electropolished samples, local defects in the form of the point protrusion develop. The RMS and mean roughness were also calculated at chosen, smaller areas free from these defects, shown as rectangles in Figs. 2 and 3. Results of such analysis is shown in Table 2.

The atomic composition of the samples was analysed by EDS mapping in SEM and shown in Fig. 4.

One of the protruding difficulties was to analyse the structure of the protrusion. Because of that the milling using Focused Ion Beam (FIB) was performed. Prior to the milling, the sample was covered with FEBID and FIBID $\mathrm{Pt}(\mathrm{C})$ material to protect the surface from being damaged during the milling [16][18]. The SEM image of the defect and the crosssection are shown in Fig. 5.

\section{Discussion}

The OP and AFM images of the samples show that the 2 min electropolishing leaves regular, scale-like residual surface pattern (Fig. 2) while the 4 min process results in smooth surface (Fig. 3). In both cases, however, local defects, in the form of point protrusions of approximated density of 100 per $100 \times 100 \mu \mathrm{m}^{2}$, develop.

The process of electropolishing reduces the evaluated surface roughness, as shown in Table 1 . The $R_{q}$ and $R_{a}$ are reduced 2.2 times and 2.6 times, respectively, for 4 min polished sample based on the AFM measurement. Unfortunately, there is a large discrepancy between the results obtained using AFM and optical profilometer, especially in RMS roughness. It may be explained by comparing the cross-sections on the profiles obtained using both these methods. These cross-sections correlate exactly witch each other on the majority of their length, apart from the protrusions which are apparently higher on the optical measure- 
ment. We believe that the true height of the protrusions is estimated using AFM.

The roughness was also estimated locally (Table 2), at the chosen smaller areas of the samples free from aforementioned protrusions. In that case, values from both optical and AFM measurements agree with each other and 4 min electropolishing process reduces the $R_{q}$ and $R_{a} 4.9$ times and 5.0 times, respectively, based on AFM measurement, and 6.2 times and 5.0 times, respectively, based on optical profilometer.

The remanium ${ }^{\circledR}$ star alloy consists of $60.5 \%$ cobalt, $28 \%$ chromium, 9\% tungsten and $1.5 \%$ silicon [2]. According to the EDS maps presented in Fig. 4, three main elements are distributed uniformly on the surface.

The SEM image of the protrusion (Fig. 5a) reveals that they are surrounded by shallow recess and characterized with quite rough top surface. The SEM image of the cross-section of the protruding defect reveals that the microstructure of the spike is the same as sample material, but characterized with nanovoids and defects (Fig. 5b).

These protrusions may, therefore, occur because of the local inclusions of the grains in the alloy which etching rate during electropolishing is smaller, or the voids in which the ion transport during the electropolishing is restricted, causing the accumulation of the etching blocking salts [19]. In both cases, in such places on the surface, the electropolishing is restricted, which causes that in their close proximity the acceptor ions concentration is higher. It may locally increase the etching rate, which would explain the observed recesses surrounding the protruding defects.

\section{Conclusions}

The untreated, raw surface of CoCrW samples are characterized by numerous recesses, which width varies from 20 up to $50 \mathrm{~nm}$ (Fig. 1). The microscopic images of the samples electropolished for 2 and 4 minutes show the progress of the process.

Presented complementary set of surface diagnostic methods enabled us to properly evaluate the surface quality of electropolished $\mathrm{CoCrW}$ alloy used for skeletal dentures. The direct comparison of atomic force microscope (AFM) and optical profilometer (OP) results showed evident discrepancies between the surface roughness values obtained using both techniques and pointing to the necessity of using more than one method to properly evaluate the surface development.

Such comparison would not be possible without using fiducial marks laser-engraved on the sample surface. They enabled us to perform both AFM and OP analysis at exactly the sample place on the sample. We observed that the OP results are influenced by the presence of sparsely located, relatively high local protrusions. These protrusions developed at the surface of the electropolished samples and they worsened the global roughness values.

One may observe that the electropolishing current imposed by the electropolishing solution manufacturer does not take the surface area of the detail being polished into account, and for typical skeletal dentures it may be higher than optimal. Nevertheless, the experiments were meant to replicate the imposed procedure and they showed the improvement of the samples' surface roughness. It is possible, however, that the results would be even better if the current was optimized.

\section{Acknowledgements}

This work was partially supported by the statutory grants of Wrocław University of Science and Technology and Wroclaw Medical University. We would like to thank Dr. Arkadiusz Antończak and Paulina Dzienny for fiducial marking fabrication. The optical profilometry measurements were conducted with kind support given by Mariusz Władowski (Optotom.pl).

\section{References}

[1] Ponto-Wolska M., Wagner L., Obróbka stopów wykorzystywanych do wykonywania protez szkieletowych. Polerowanie elektrochemiczne $w$ wybranych elektrolitach, Protet. Stomatol., 2014, 64, 354-360.

[2] Dentaurum, remanium ${ }^{\circledR}$ star - Product information, 2019.

[3] Ponnanna A.A., Joshi S.M., BhAt S., ShetTy P., Evaluation of the polished surface characteristic of cobalt-chrome castings subsequent to various finishing and polishing techniques, Indian J. Dent. Res., 2001, 12, 222-228.

[4] Zinelis S., Thomas A., Syres K., Silikas N., Eliades G., Surface characterization of zirconia dental implants, Dent. Mater., 2010, 26, 295-305.

[5] Angelini E., ZuCCHi F., In vitro corrosion of some Co-Cr and $\mathrm{Ni}-\mathrm{Cr}$ alloys used for removable partial dentures: influence of heat treatments, J. Mater. Sci. Mater. Med., 1991, 2, $27-35$.

[6] Sinclair G.F., RAdford D.R., Sheriff M., Walter J.D., Effects of Electrobrightening on the Fit Surface of Cobalt-Chromium RPD Frameworks, Int. J. Prosthodont., 2000, 13, 232-237.

[7] ŢĂLu S., Stach S., Klaić B., MišIĆ T., Malina J., Čelebić A., Morphology of Co-Cr-Mo dental alloy surfaces polished by three different mechanical procedures, Microsc. Res. Tech., 2015, 78, 831-839.

[8] Jalili N., Laxminarayana K., A review of atomic force microscopy imaging systems: Application to molecular metrology and biological sciences, Mechatronics, 2004, 14, 907-945. 
[9] Sharma S., Cross S.E., Hsueh C., Wali R.P., Stieg A.Z., GIMZEWSKI J.K., Nanocharacterization in dentistry, Int. J. Mol. Sci., 2010, 11, 2523-2545.

[10] Sivel V.G.M., Van Den Brand J., Wang W.R., Mohdadi H., TichelaAR F.D., AlKemade P.F.A., ZANDBERGEN H.W., Application of the dual-beam FIB/SEM to metals research, J. Microsc., 2004, 214, 237-245.

[11] Ritter M., Dziomba T., Kranzmann A., Koenders L., A landmark-based $3 D$ calibration strategy for SPM, Meas. Sci. Technol., 2007, 18, 404-414.

[12] http://www.chema.rzeszow.pl/produkt/elektrol-11.html, 2019.

[13] Bhushan B., Nanotribology and nanomechanics, second edition, An introduction, 2008.

[14] NeČAS D., KLAPETEK P., Gwyddion: An open-source software for SPM data analysis, Cent. Eur. J. Phys., 2012, 10, 181-188.
[15] KlapeteK P., Quantitative Data Processing in Scanning Probe Microscopy: SPM, Applications for Nanometrology, 2012.

[16] Singh D.R.P., Chawla N., Shen Y.-L., Focused Ion Beam (FIB) tomography of nanoindentation damage in nanoscale metal/ ceramic multilayers, Mater. Charact., 2010, 61, 481-488.

[17] INKSON B.J., STEER T., MÖBUS G., WAGNER T., Subsurface nanoindentation deformation of $\mathrm{Cu}$-Al multilayers mapped in $3 \mathrm{D}$ by focused ion beam microscopy, J. Microsc., 2001, 201, 256-269.

[18] Winiarski J., Cieślikowska B., Tylus W., Kunicki P., SzCZYGiEx B., Corrosion of nanocrystalline nickel coatings electrodeposited from choline chloride: ethylene glycol deep eutectic solvent exposed in $0.05 \mathrm{M} \mathrm{NaCl}$ solution, Appl. Surf. Sci., 2019, 470, 331-339.

[19] Yang G., Wang B., TawfiQ K., Wei H., Zhou S., Chen G., Electropolishing of surfaces: theory and applications, Surf. Eng., 2017, 33, 149-166. 